\title{
Incremental, scalable tracking of objects inter camera
}

\author{
Andrew Gilbert and Richard Bowden \\ CVSSP, University of Surrey, Guildford, GU2 7XH, England
}

\begin{abstract}
This paper presents a scaleable solution to the problem of tracking objects across spatially separated, uncalibrated cameras with non overlapping fields of view. The approach relies on the three cues of colour, relative size and movement between cameras to describe the relationship of objects between cameras. This relationship weights the observation likelihood for correlating or tracking objects between cameras. Any individual cue alone has poor performance, but when fused together, a large boost in accuracy is gained. Unlike previous work, this paper uses an incremental technique to learning. The three cues are learnt in parallel and then fused together to track objects across the spatially separated cameras. The colour appearance cue is incrementally calibrated through transformation matrices, while probabilistic links, modelling an object's bounding box, between cameras represent the objects relative size. Probabilistic region links between entry and exit areas on cameras provide the cue of movement. The approach needs no pre colour or environment calibration and does not use batch processing. It works completely unsupervised, and is able to become more accurate over time as new evidence is accumulated.
\end{abstract}

\section{Introduction}

Surveillance cameras are increasingly being used as a tool to monitor and deter crime. As a result, there are large numbers of cameras which lack effective continuous monitoring due to the limitations of humans in managing largescale systems. Therefore, tools to assist and aid the operator's decision process are essential. The approach presented in this paper aims to automatically track objects between the cameras (inter camera). This is termed "object handover", where one camera transfers a tracked object or person to another camera. To

Email address: a.gilbert, r.bowden@surrey.ac.uk (Andrew Gilbert and Richard Bowden). 
be able to achieve successful object handover we need to learn about the environment in which the cameras operate. However, the system needs to be able to achieve this without a priori information i.e. no colour, spatial or environmental calibration. In fact, as cameras may have no overlapping field of view, many traditional calibration techniques are impossible. An ideal tracking system could be described by the following:

- It is able to work immediately upon initialisation,

- Performance will improve as new evidence becomes available,

- Is adaptable to changes in the camera's environment

To be able to fulfil these aims the system needs to learn the relationships between the non-overlapping cameras automatically. Here we achieve this by modelling colour, relative size and movement. The three cues are deliberately very weak as more detailed and complex cues would not be able to work with the low resolution and real time requirements of the system. These three weak cues, are then fused together to allow the system to determine if objects have been previously tracked on another camera or are new to the system. The approach learns these camera relationships, though unlike previous work does not require a priori calibration or explicit training periods. The use of incremental learning of these cues allows for the accuracy to increase over time without supervised input. Preliminary work was presented in [10], this manuscript builds on our previous work to present a novel approach to inter camera tracking which fuses additional features with a scalable architecture providing accurate object handover between cameras. We demonstrate performance with extensive experimental testing and results and compare our incremental learning with traditional batch approaches.

The paper firstly describes background material relevant to inter camera tracking and calibration. Then an overview of the system is presented, and the intra camera tracking technique is explained in section 4. Learning of probabilistic region links is described in section 5 , followed by how the objects size can also be used in section 6 . Section 7 then explains calibrating the inter camera colour response incrementally, before the results are presented in section 8. A discussion of system scalability and conclusion are given in sections 9 and 10 respectively.

\section{Related Work}

Early tracking algorithms [4][16] required both camera calibration and overlapping fields of view (FOV). These were needed to compute the handover of tracked objects between cameras. Additionally, Chang [5] required a 3D 
model of the environment using epipolar geometry, to allow for the registration of objects across the different overlapping cameras. The requirement that cameras have an overlapping FOV is impractical due to the large number of cameras required and the physical constraints upon their placement.

Kettnaker and Zabih [17] presented a Bayesian solution to tracking people across cameras with non-overlapping FOVs. However the system required calibration, with the user providing a set of transition probabilities and their expected duration a priori. This means that the environment and the way people move within it must be known. In most surveillance situations this is unrealistic.

Probabilistic or statistical methods have recently seen some of the greatest focus in solving inter camera tracking. They all use the underlying principle that through accumulating evidence of movement patterns over time, it is likely that common activities will be discovered. Huang and Russel [11] presented a probabilistic approach to tracking cars on a highway, modelling the colour appearance and transition times as gaussian distributions. This approach is very application specific, using only two calibrated cameras, with vehicles moving in one direction in a single lane. Javed et al. [13] present a more general system by learning the camera topology and path probabilities of objects using Parzen windows. This is a supervised learning technique where transition probabilities are learnt during training using a small number of manually labelled trajectories. Dick and Brooks [6] use a stochastic transition matrix to describe patterns of motion both intra and inter camera. The system required an offline training period where a marker was carried around the environment. This would be infeasible for large systems and can not adapt to cameras being removed or added ad hoc without recalibration. For both systems [13] [6], the correspondence between cameras has to be supplied as training data a priori.

KaewTraKulPong and Bowden [14] or Ellis et al. [8] do not require a priori correspondences to be explicitly stated, instead they use the observed motion over time to establish reappearance periods. Ellis learns the links between cameras, using a large number of observed objects to form reappearance period histograms between the cameras. KaewTraKulPong uses appearance matching to build up fuzzy histograms of the reappearance period between cameras. This allows a spatio-temporal reappearance period to be modelled. In both cases batch processing was performed on the data which limits their application to the real world. 
Colour is often used in the matching process. Black et al. [2] use a non-uniform quantisation of the HSI colour space to improve illumination invariance, while retaining colour detail. KaewTraKulPong and Bowden [15] uses a ConsensusColour Conversion of Munsell colour space (CCCM) as proposed by Sturgeset al. [22]. This is a coarse quantisation based on human perception and provides consistent colour representation inter-camera. Most multi camera surveillance systems assume a common camera colour response. However, even cameras of the same type will exhibit differences which can cause significant colour errors. Pre-calibration of the cameras is normally performed with respect to a single known object, such as the GretagMacbeth [20] ColorCheckerTM chart with twenty four primary colours used by Ilie and Welch [12]. Porikli [19] proposes a distance metric and model function to evaluate the inter camera colour response. It is based on a correlation matrix computed from three 1-D quantised RGB colour histograms and a model function obtained from the minimum cost path traced within the correlation matrix. Joshi [18] similarly proposes a RGB to RGB transform between images. By using a 3x3 matrix, inter channel effects can be modelled between the red, green, and blue components. Annesley and Orwell [1] model colour variation between cameras to enforce colour consistency between cameras, using the grey-world assumption to model the colour variation.

\section{Experiment Setup and System Overview}

The experimental setup consists of 4 colour cameras with non-overlapping fields of view in an indoor office environment, with the layout shown in figure 1. The areas not visible between cameras contain doors and corners ensuring no Straight-line trajectories or linear velocities are possible between cameras. The four time synchronised video feeds are fed into a $\mathrm{P} 4$ Windows $\mathrm{PC}$ in real-time. Figure 2 gives a general overview of the system. This figure shows an example
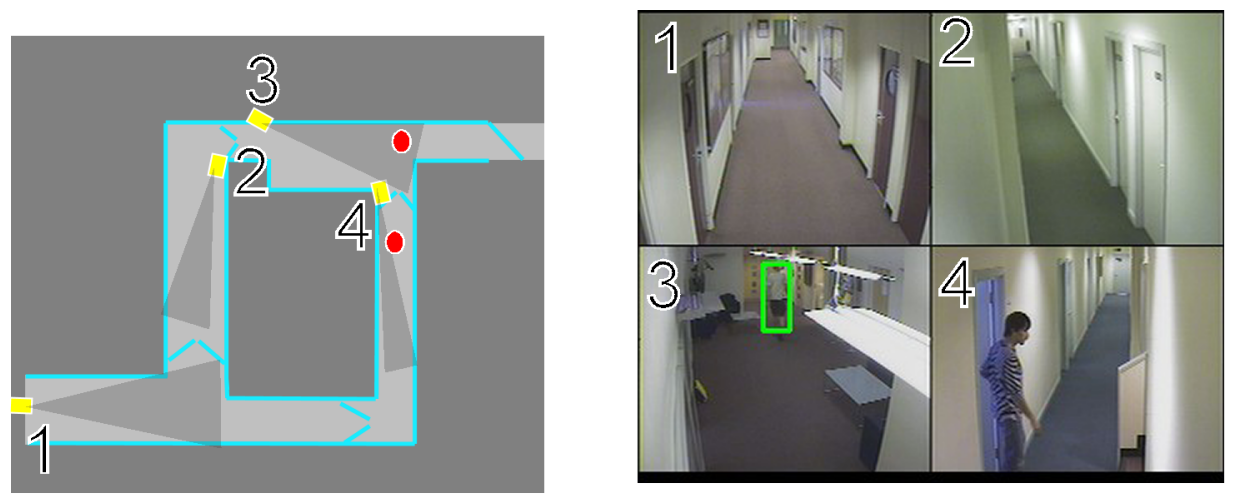

Fig. 1. (Left)The top down layout of the camera system and areas visible to the cameras, (Right) An example image of the tracking environment used. 
containing two camera modules and a module for the operator to query the cameras about objects. Each camera is a self contained module connected to others via a network, meaning that it can easily be distributed over multiple processors or machines. An overview of each stage is given below:

- Object Detection The camera image is fed into an object detection module where a background scene model is maintained and updated. This model is used to delineate foreground from background for the incoming image.

- Intra Camera Tracking Foreground objects are correlated to objects in the previous frame using a Kalman filter to provide intra camera object tracking. If a correlation with an object in the previous frame is found, the object is labelled as an Old Object and the colour descriptor for that object is updated. If no correlation exists, it is labelled as a New Object and if an object from the previous frame has no correlation to any object in the current frame, it is deemed an Exiting Object. The Kalman filter continues positional predictions for the Exiting Object to overcome object occlusions, but after a set time with no incoming correlation, it is deemed to have left the camera. At this point, the Exiting Object's colour, size, and position descriptor are broadcast via the network to all other camera modules to enable inter camera tracking.

- Inter Camera Tracking When an object is labelled a New Object, its descriptor is compared to objects that have previously exited other cameras and been broadcast as potential candidates for object handover. This comparison is based upon colour similarity weighted by the prior models of how cue relate between cameras.

- Update System Cues If a potential object handover is identified, the object's colour similarity is used to provide a weighted update to camera colour calibration matrices, the relative size of the bounding box, and iterative region linking scheme.

In this way each camera maintains a model of how other cameras in the network relate to it. When an operator wishes to track a specific object over the camera network, a request is sent via the network to query all cameras and possible matches are returned to the operator.

\section{Intra Camera Object Tracking and Description}

To detect moving objects, the static background is modelled in a similar fashion to that originally presented by Stauffer and Grimson [21]. The foreground vs background pixel segmentation is formed using a gaussian mixture model on a per pixel basis [14]. The mixtures represent the probability of background, with the pixel history modelled using a weighted mixture of Gaussians. The weight is proportional to the number of observations that a component gaus- 


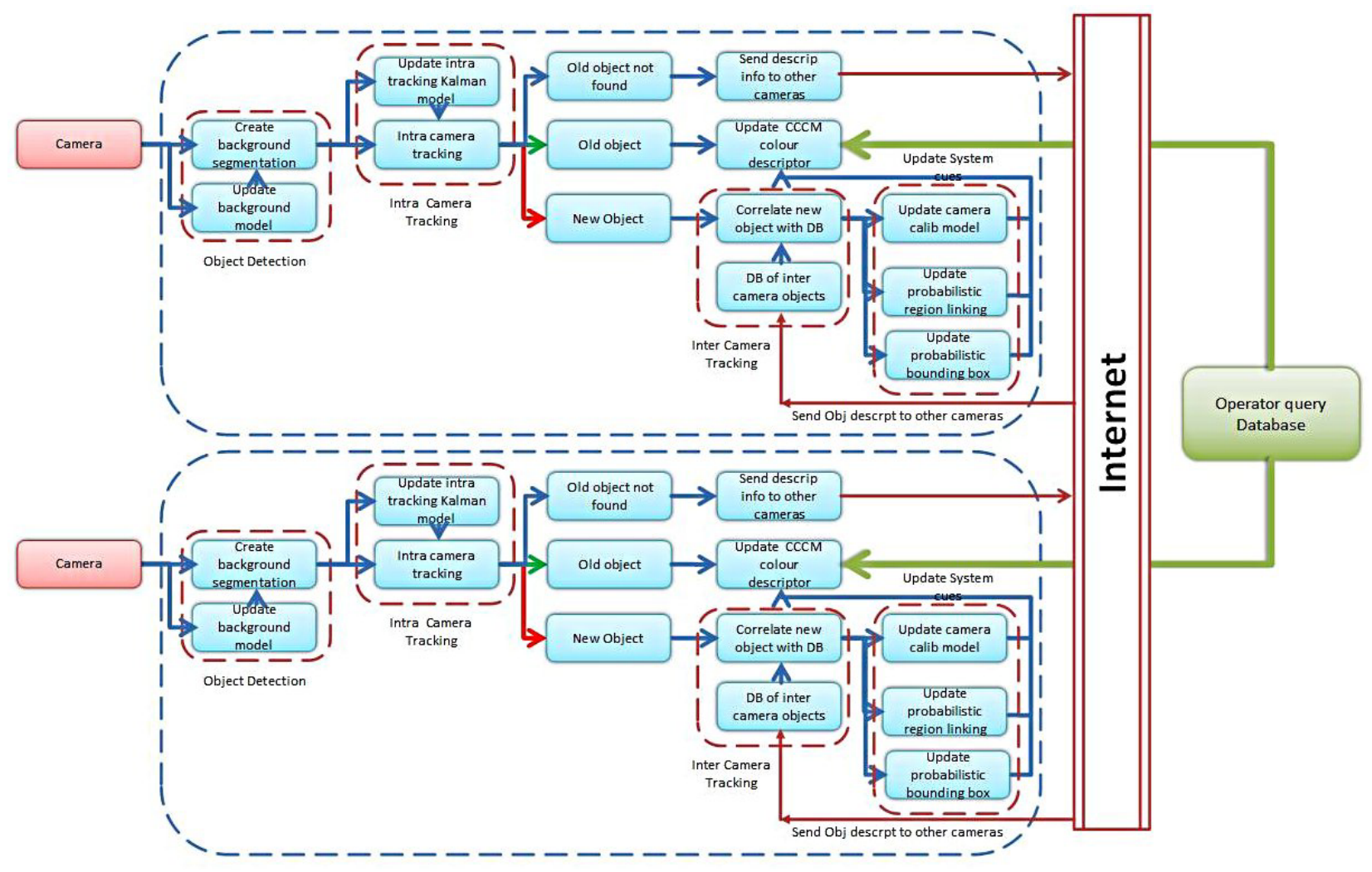

Fig. 2. System Overview with two independent camera tracking modules connected together by a network. With an operator query module for tracking a specific object inter camera

sian represents. For each new frame, all pixel values are compared to the existing model components. If a correlation is found, the pixel is set as background and the component mean and variance adjusted accordingly. If no match is found a new gaussian component is formed and the weights amended appropriately. Shadows are identified and removed by relaxing a models constraint on intensity, and the foreground object is formed using connected component analysis on the resulting binary segmentation. The advantage of this background segmentation method is that when a new object appears in the scene, it is only classified as background if it is stationary for a long period of time, while the existing background model is not destroyed. Once the object moves, the distribution describing the previous background still exists (with a lower weighting), and will be quickly regain dominance in the model. The foreground objects are linked temporally with a Kalman filter providing smooth motion trajectories within each (intra) camera, illustrated in figure 1. The Kalman filters are updated using the standard Kalman filter update rules [24] using a constant velocity model with a white noise drift term. The Kalman filter estimates are represented by the mean and covariance of the centre of an object's bounding box. 


\subsection{Object Appearance Modelling}

Once the foreground objects have been segmented, a colour descriptor can be formed to provide the model of that object. The colour histogram is used to describe the objects appearance as it is invariant to scale and the spatial distribution of the pixels which change dramatically between different cameras. Through colour space quantisation some level of illumination invariance can be introduced. Several colour spaces and quantisation levels were investigated including the HSI quantisation (8x8x4) approach proposed by Black et al. [2], the Consensus-Colour Conversion of Munsell colour space (CCCM) [22] and differing levels of traditional RGB quantisation. Without calibrating camera colour responses, CCCM produced marginally superior results and was selected for initial object correlation, for further details see [3]. CCCM works by breaking the RGB colour space into 11 discrete colours. Each basic colour represents a perceptual colour category established through a physiological study of how human's categorise colour. This coarse quantisation provides a consistent colour representation inter-camera prior to calibration. However, if the cameras are colour calibrated, CCCM discards too much discriminatory information through its coarse quantisation, so once the initial unsupervised colour calibration has taken place (see section 7) quantised RGB is used. However, the RGB quantisation is very sensitive to the number of bins selected, to overcome this, a Parzen window is used.

\subsection{Parzen windowing the $R G B$ colour space}

There are two types of Parzen Windowing, Post-Parzen Windowing and InParzen Windowing [7]. Post-Parzen Windowing involves convolving the resultant colour quantisation histogram with a kernel, often a gaussian. This is a computationally cheap method of smoothing, however, it results in a loss of information due to the application of the gaussian after the quantisation has taken place. In-Parzen Windowing is designed to reduce the effect of different quantisation levels by convolving each sample with a kernel prior to quantisation then populating multiple bins in the colour histogram. Although performing In-Parzen Windowing during the histogram construction is comparatively expensive, and some loss of information still occurs, in-Parzen Windowing produces a more accurate histogram compared to Post-Parzen Windowing. Therefore, RGB quantisation with In-Parzen Windowing is used for the final object appearance models discussed in Section 7 . 


\section{$5 \quad$ Probabilistic inter camera coupling}

A simple quantisation of colour alone cannot describe objects sufficiently inter camera due to illumination variations, and occlusions. Therefore, we propose three methods to learn the relationships between the cameras. The first method tries to incrementally learn the probabilistic relationship of object movements between cameras. This makes use of the key assumption that, given time, objects (such as people or cars) will follow similar routes (inter camera) due to paths, shortest routes and obstructions. The repetition of these routes over time, will then start to form marked trends in the data. We model the reappearance period between two cameras by calculating the probability that an object disappearing from one camera at time zero, will reappear in another camera at time $t$. This allows us to link common entry and exit regions between cameras. The links, modelled as conditional probabilities, are constructed using histograms populated over time as evidence is gathered. As the number of possible links increase, so does the quantity of data required to populate the histograms. However, most links are invalid as they correspond to impossible routes, such as entry points on walls, or between cameras too distant to be reliable. Thus we propose a method to identify the valid and invalid links without user supervision. Previous solutions required either batch processing or hand labelling to identify entry/exit points, both impractical in large systems, and unable to adjust to camera or environmental changes. Our approach is initially coarsely defined, but increases in detail over time as more evidence becomes available, and can adjust to changes without a system restart.

\subsection{Incremental link learning}

Objects are automatically tracked intra camera with a Kalman filter to form a colour appearance model of the object. The CCCM colour histogram $B=$ $\left(b_{1}, b_{2} \ldots b_{n}\right)$ is the median histogram recorded for an object over its entire trajectory within a single camera. All new objects that are detected are compared to previous objects exiting other cameras within a set time window, $T$ (See section 8 for a discussion of $T$ ). For each region-to-region link a discrete probability distribution is formed of the colour correlation of new objects with respect to their reappearance period $T$. The colour correlation is computed using histogram intersection [23], other correlation measures such as Bhattacharyya distance and the $c h i^{2}$ test were examined, but due to the coarseness of colour quantisation they give no discernible difference in results. Thus the frequency 
$f$ of a bin $\phi$ of a histogram representing reappearance periods is calculated as

$$
f_{\phi}=\sum_{\forall i} \sum_{\forall j}\left\{\begin{array}{cl}
H_{i j} & \phi \mu \leq\left(t_{i}^{\text {end }}-t_{j}^{\text {start }}\right)<(\phi+1) \mu \\
0 & \text { otherwise }
\end{array} \quad \forall \phi, \phi \mu<T\right.
$$

where $t_{i}^{\text {start }}$ and $t_{i}^{\text {end }}$ are the entry and exit times of object $i$ respectively, $T$ is the maximum allowable reappearance period and $\mu$ is the bin size in seconds. $H_{i j}$, the histogram intersection of objects $i$ and $j$ is calculated as

$$
H_{i j}=\sum_{k=1}^{11} \min \left(B_{i k}, B_{j k}\right)
$$

For In-Parzen Windowing equation 1 becomes

$$
f_{\phi}=\sum_{\forall i} \sum_{\forall j} H_{i j} G\left(t_{i}^{\text {end }}-t_{j}^{\text {start }}-\phi \mu\right) \quad \forall \phi, \phi \mu<T
$$

where $G(t)$ is a $1 \mathrm{D}$ gaussian with variance $\sigma^{2}$

$$
G(t)=\frac{1}{\sigma \sqrt{2 \pi}} e^{-\frac{t^{2}}{2 \sigma^{2}}}
$$

Frequencies are only calculated for an object $i$ that disappears from region $y$ followed by a reappearance in region $x\left(f^{x \mid y}\right)$. By normalising the total area of the histogram by $\sum_{i}^{T} f_{\phi}^{x \mid y}$, an estimate to the conditional transition probability $P\left(O_{x, t} \mid O_{y}\right)$ is obtained. An example of $P\left(O_{x, t} \mid O_{y}\right)$ is shown in Figure 3 where $O_{x, t}$ is object $x$ at time $t$. This probability distribution shows a distinct peak at 9 seconds indicating a link between cameras 1 and 4 with a single region per camera.

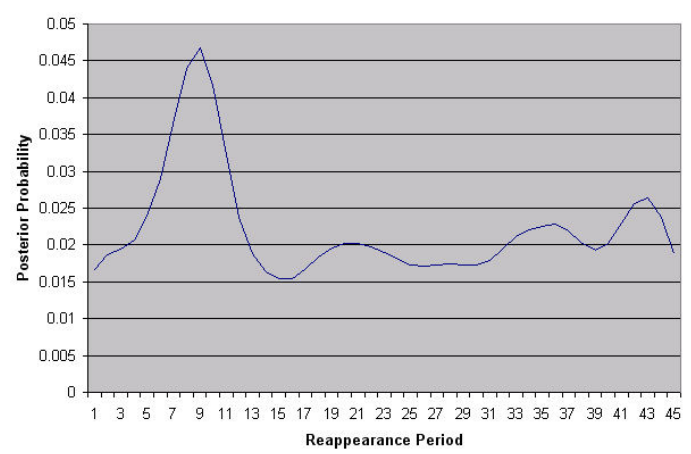

Fig. 3. An example of a probability distribution showing a distinct link between cameras 1 and 4 with a single region per camera over a reappearance period of 45 seconds 
After sufficient evidence has been accumulated, determined by the degree of histogram population, the noise floor level is measured for each link. This could be determined statically using the mean or variance, however, though experimentation, using double the median of histogram values was found to provide more consistent results. Figure 4 shows how the reappearance period of objects between cameras 3 and 2 of figure 1 develops as observations are added over time. A peak reappearance probability at around 10 seconds increases in height as people are tracked and evidence is added to the distribution. After 1000 people have been accumulated there is a distinct peak around a reappearance period of 10 seconds. These probabilistic temporal inter camera

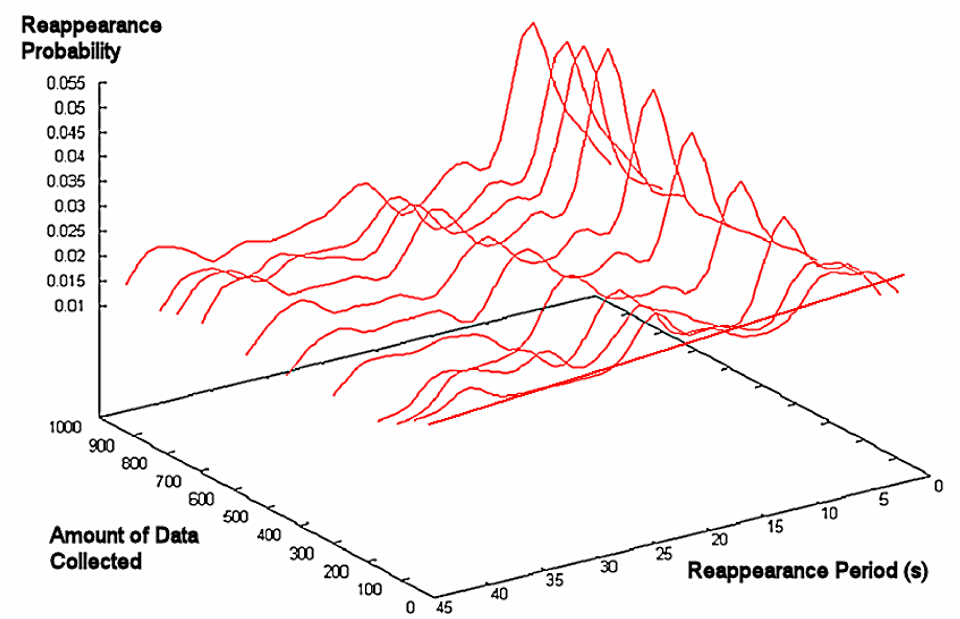

Fig. 4. The reappearance period probability between camera 3 and 2 as more data is collected over 1 day

links can be used to couple camera regions together, producing a probabilistic distribution of an objects movement between cameras. If the maximum peak of the distribution is found to exceed the noise floor level, this indicates a possible coupling between the regions. If a possible coupling has been found, the linked parent regions are subdivided into four child regions as in figure 5 . The initial distributions of the four new regions are set to that of the parent. Subsequent data is incorporated into the appropriate refined distribution.

In order to allow for multiple entry and exit area on the cameras, each camera is split into a number of equal regions, 16 on the current system. Coupling all regions to all others is only feasible in small-scale experimental systems. As the number of cameras increase, the number of links required to model the prior will increase exponentially. With 16 regions between 2 cameras, there are $2^{16}$ (65536) links, with just 2 more cameras this becomes $4^{16}$ (4294967296) links. However, many regions will not form coherent links, and can therefore be removed to minimise the number maintained. It is important that links 
are not removed between regions that simply require more data, therefore, a link between two regions is only removed if it has no data in it at all. This cautious method ensures no regions or links are removed that might be useful in a later subdivision.

Figure 5 shows how the active regions are sub divided or removed over time. At system start-up there are no regions as shown in initial start-up, each camera is a single region, with a uniform conditional probability of objects moving between cameras. After the first 367 tracked objects, subdivision 1 shows how the cameras are linked together. Subdivision 2 occurs after 1372 objects have been added to the system and unlinked regions are removed. Further subdivision and removal of regions is achieved in subdivision 3 following 2694 objects and subdivision 4 at 7854 objects. The remaining regions in subdivision 4 show the main entry and exit areas. Table 1 shows the number of links main-
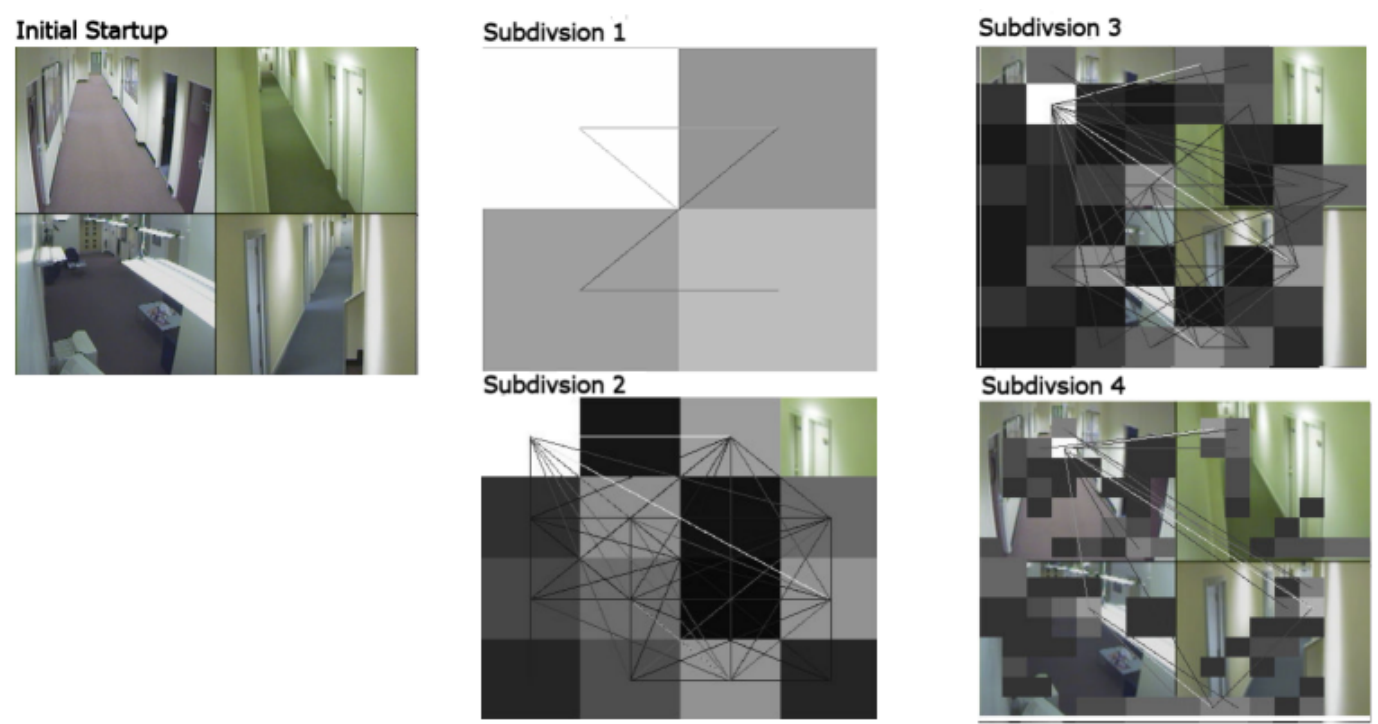

Fig. 5. The iterative process of splitting the blocks on the video sequence over a day

tained and dropped at each subdivision stage, along with the amount of data used. It can be seen that with each iteration, the number of possible links increases dramatically, whereas the number of valid links kept is considerably less. The policy of removing unused and invalid regions improves the systems scalability. This iterative process can be repeated to further increase the resolution of the blocks. The regions start to form the entry and exit points of the cameras, figure 6 (left image) shows the result after 4 subdivisions. The lighter regions have a higher importance determined by the number of samples each link contains. As the number of iterations increase, the size of the linked regions decreases and thus reduces the number of samples detected in each region. This affects the overall reliability of the data used. To counter this, regions which are found to have similar distributions to neighbouring regions are combined together to increase the overall number of samples within the 


\begin{tabular}{|c|c|c|c|c|c|c|}
\hline Iteration & $\begin{array}{c}\text { Amount of } \\
\text { Data }\end{array}$ & $\begin{array}{c}\text { Number of } \\
\text { Regions }\end{array}$ & $\begin{array}{c}\text { Tot poss } \\
\text { Links }\end{array}$ & $\begin{array}{c}\text { Initial } \\
\text { links }\end{array}$ & $\begin{array}{c}\text { Dropped } \\
\text { links }\end{array}$ & Kept links \\
\hline 1 & 367 & 4 & 12 & 12 & 0 & 12 \\
2 & 1372 & 16 & 240 & 240 & 45 & 195 \\
3 & 2694 & 60 & 2540 & 1631 & 688 & 943 \\
4 & 7854 & 191 & 36290 & 36134 & 34440 & 1694 \\
\hline
\end{tabular}

Table 1

Table of number of links maintained and dropped in each iteration of region subdivision
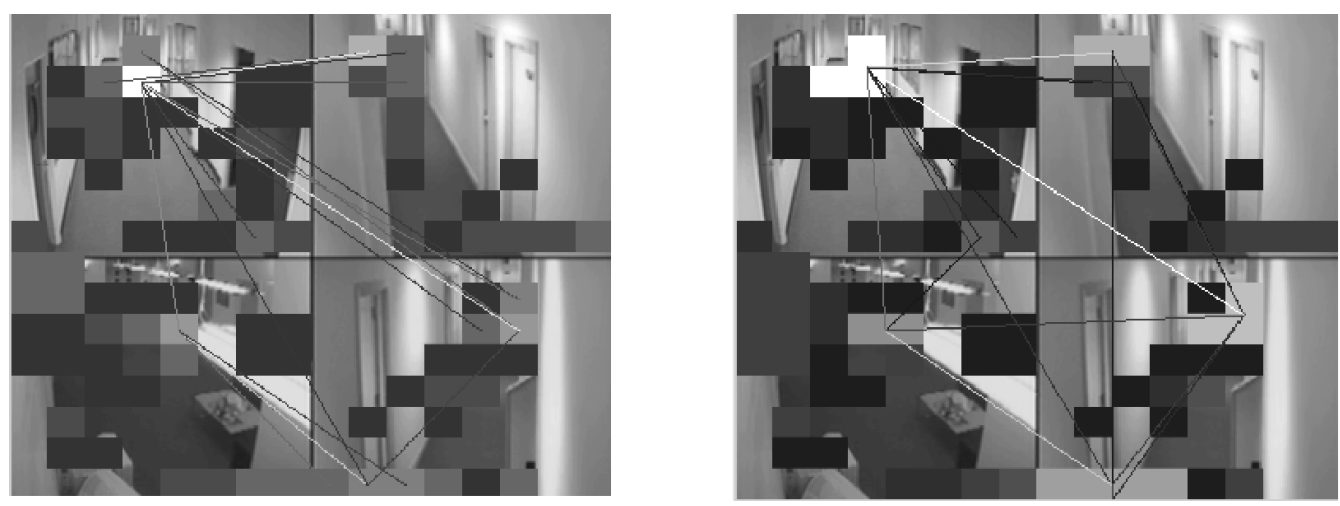

Fig. 6. Left image shows the main identified entry/ exit regions. Right Image shows the individual regions that, if similar, are then recombined to form larger more populated regions, shown by the constant colour areas.

region (as illustrated in the right image in figure 6). This reduces the overall number of regions maintained and the actual links between regions, therefore increasing the accuracy of the remaining links.

\subsection{Calculating Posterior Appearance Distributions}

The conditional prior probability of objects moving between cameras can be used to weight the observation of tracked people providing a posterior probability that an object has moved between regions. Over time the prior and therefore the posterior becomes more accurate as the subdivision process (previously described) takes place. Given an object which disappears in region $y$ we can model its reappearance probability over time as;

$$
P\left(O_{t} \mid O_{y}\right)=\sum_{\forall x} w_{x} P\left(O_{x, t} \mid O_{y}\right)
$$


where the weight $w_{x}$ at time $t$ is given as

$$
w_{x}=\frac{\sum_{i=0}^{T} f_{\phi}^{x \mid y}}{\sum_{\forall y} \sum_{i=0}^{T} f_{\phi}^{x \mid y}}
$$

This probability is then used to weight the observation likelihood obtained through colour similarity to obtain a posterior probability of a match, across spatially separated cameras. Bayes provides a method to estimate the posterior.

$$
P(A \mid B)=\frac{P(B \mid A) P(A)}{P(B)}
$$

where $P(B \mid A)$ is our prior conditional probability $P\left(O_{t} \mid O_{y}\right)$ from equation 5 and $P(A)$ our observation likelihood $H_{i j}$. Thus the posterior for a newly detected object $x$ being object $y$ at time $t$ can be given by

$$
P\left(O_{y} \mid O_{x}\right)=\text { observation } * \text { prior }=H_{i j} * P\left(O_{x, t} \mid O_{y}\right)
$$

Tracking of objects is then achieved by maximising the posterior probability within a set time window. A detailed discussion is deferred to section 8 .

\section{Probabilistic Inter Camera Bounding Box}

The background segmentation used, provides a background mask, with the foreground objects labelled via connected component analysis. Around each of the detected objects a bounding box is formed based on the mean and standard deviation of the blob in pixels, figure 7 shows the rectangular bounding box of a person. The size of this bounding box provides a coarse size descriptor

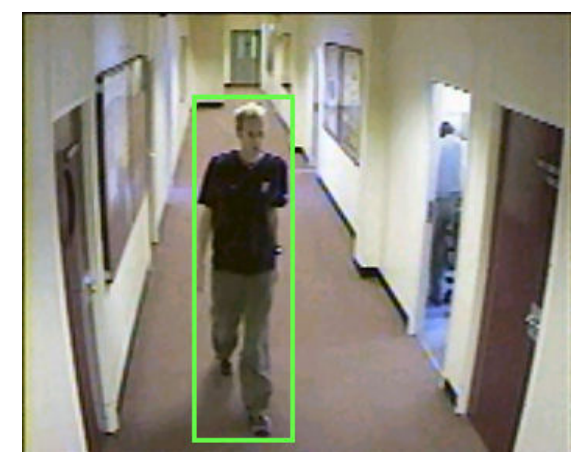

Fig. 7. The rectangular bounding box around a detected object

of the object in the image plane as it moves upon the ground plane. Objects 
further from the camera will have a smaller bounding box size, with closer objects having a larger size. As in the previous section we can assume that, over time, objects follow similar routes inter camera. This means that they will leave and enter cameras in consistent areas and therefore the size of the object should be consistent upon entry or exit. Looking at the experimental environment in figure 2 , if a person moves between camera 4 and 3 , they will leave camera 4 at the bottom of the camera with a large bounding box, and should reappear towards the top of camera 3 with a relatively small bounding box. This fact can be utilised to calculate the likelihood that a person has come from another camera based upon the relative entry size to the current camera.

The relationship between the exit size from one camera and the entry size in another can be represented by a 3D histogram, but due to the problems associated with having sufficient observations to populate such a histogram we assume independence and model the relationship as two 2D histograms. Figure 8 shows this relationship for the $\mathrm{x}$ size of the bounding box between cameras 1 and 4 . These relationships are only modelled at a camera-to-camera

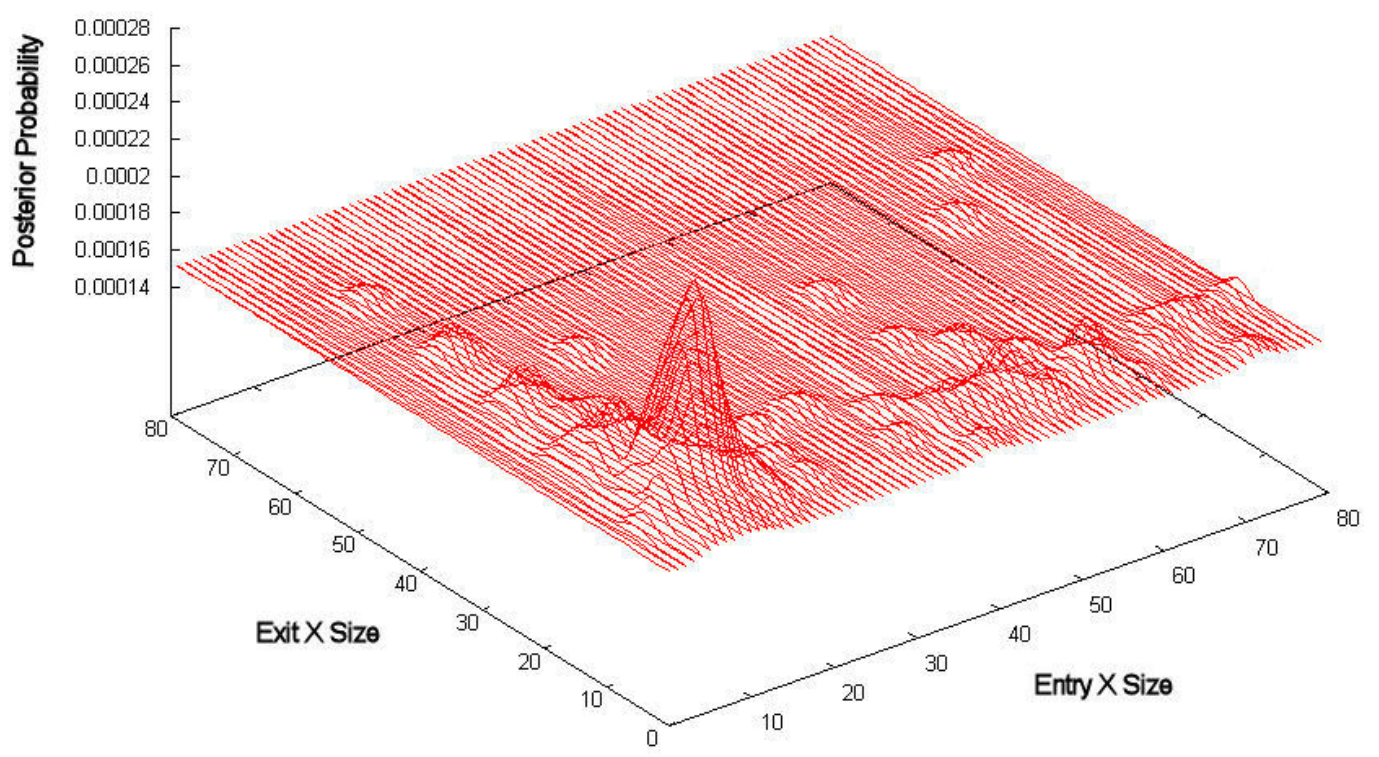

Fig. 8. The probability distribution of the $\mathrm{x}$ axis bounding box between cameras 1 and 4

level and there are therefore 24 discrete histograms which link all 4 camera to one another. The 2D distributions are learnt over time in a similar way to the probabilistic spatial temporal coupling in the previous section. The histograms all start uniformly distributed. All new objects are compared to previous objects within the reappearance period $T$. The match score is computed using the histogram intersection formula in equation 2 . The new object $j$ will have an entry size and the old object $i$ an exit size, so the observation is then used 
to increment the appropriate bin in the 2D histogram for the specific link by the strength of the correlation (colour similarity). Thus the frequency of a bin for the $\mathrm{x}$ axis between two the cameras $i$ and $j$ with the bounding box, size, is calculated as

$$
f\left(\operatorname{size}_{i}^{e x i t}, \operatorname{size}_{j}^{\text {entry }}\right)_{x}=\sum_{\forall i} \sum_{\forall j} H_{i j} * G\left(s i z e_{i}^{e x i t}, \operatorname{siz}_{j}^{\text {entry }}\right)\left(t_{i}^{\text {end }}-t_{j}^{\text {start }}\right)<T(9)
$$

where $H_{i j}$ is the histogram intersection between the objects from equation 2 and $G$ is a $2 \mathrm{D}$ gaussian defined with spherical variance $\left(\sigma^{2}\right)$ as

$$
G(x, y)=\frac{1}{2 \pi \sigma^{2} \sigma^{2}} e^{-\left[\frac{\left(x-\mu_{x}\right)^{2}}{2 \sigma^{2}}+\frac{\left(y-\mu_{y}\right)^{2}}{2 \sigma^{2}}\right]}
$$

$f(\alpha, \beta)_{x}$ is then normalised, by the total area of the distribution to provide the conditional probability of the resulting change in bounding box size $P\left(O_{x, \text { Entry }} \mid O_{y, \text { Exit }}\right)$ where $O_{x, \text { Entry }}$ is object $x$ with an entry size of entry. Over time, the prior of the size of entry and exit bounding box will become more accurate as more data is collected. This is then used to weight the observation likelihood obtained through colour similarity as was done in the previous section. As will be seen in section 8 , these cues can be combined by simply multiplying their likelihoods together.

\section{Inter Camera Colour Calibration}

The CCCM colour quantisation descriptor used in the previous sections assumes a similar colour response between cameras. However this is seldom the case, the cameras of Figure 1 show a marked difference in colour response even to the human eye. Therefore, a colour calibration of these cameras is proposed that can be learnt incrementally as with the distributions previously discussed.

The system initially uses the CCCM colour descriptor to form posterior distributions. Once sufficient colour calibration is achieved, a traditional RGB quantisation with In-Parzen Windowing is used. The colour transformation matrices between cameras are constructed in parallel with the construction of priors on reappearance probability and size. The tracked people are automatically used as the calibration objects, and as shown in figure 9, a transformation matrix is formed incrementally to model the colour changes between cameras. As people vary in size, a point to point transformation is unavailable. We therefore use the colour descriptor (a histogram) of the object in the different cameras to provide the calibration. As our histograms represent the probability distribution of an objects colour within a camera, a simple linear transform 


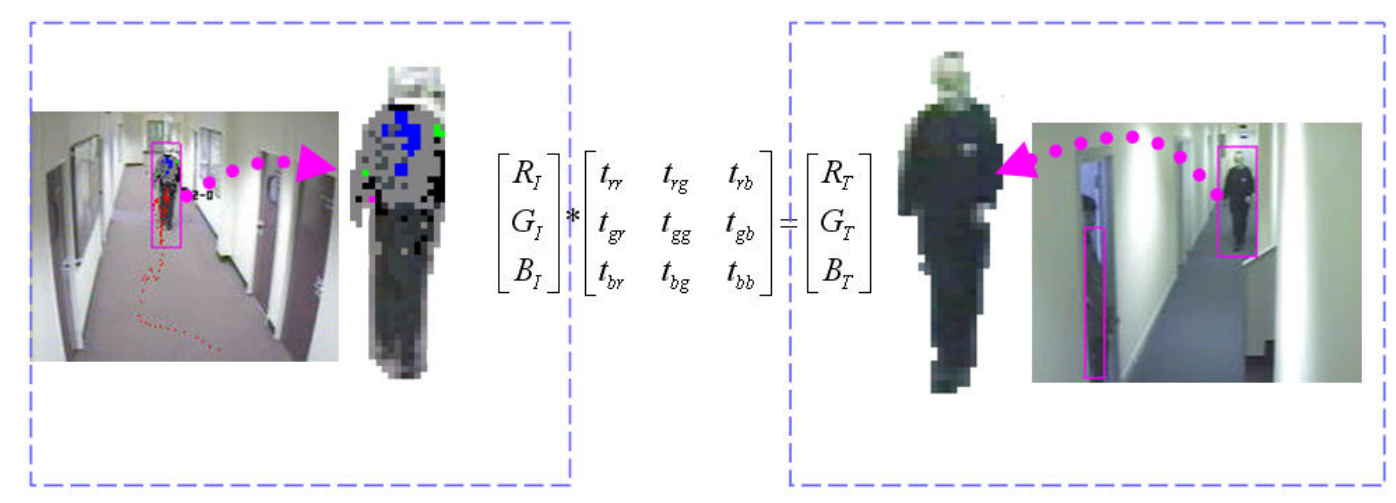

Fig. 9. An illustration of when the transform matrix is used

is capable of providing the pdf to pdf mapping [9]. For our work we use a 6 bin RGB histogram quantisation, however for illustration, Figure 10 shows the transformation matrix between image $I$ and the transformed image $T$ using a simple 2 bin RGB quantisation.

$$
\left[\begin{array}{llllll}
I_{r_{1}} & I_{r_{2}} & I_{g_{1}} & I_{g_{2}} & I_{b_{1}} & I_{b_{2}}
\end{array}\right] *\left[\begin{array}{cccccc}
t_{r_{1} r_{1}} & t_{r_{1} r_{2}} & t_{r_{1} g_{1}} & t_{r_{1} g_{2}} & t_{r_{1} b_{1}} & t_{r_{1} b_{2}} \\
t_{r_{2} r_{1}} & t_{r_{2} r_{2}} & t_{r_{2} g_{1}} & t_{r_{2} g_{2}} & t_{r_{2} b_{1}} & t_{r_{2} b_{2}} \\
t_{g_{1} r_{1}} & t_{g_{1} r_{2}} & t_{g_{1} g_{1}} & t_{g_{1} g_{2}} & t_{g_{1} b_{1}} & t_{g_{1} b_{2}} \\
t_{g_{22} r_{1}} & t_{g_{2} r_{2}} & t_{g_{2} g_{1}} & t_{g_{2} g_{2}} & t_{g_{2} b_{1}} & t_{g_{2} b_{2}} \\
t_{b_{1} r_{1}} & t_{b_{1} r_{2}} & t_{b_{1} g_{1}} & t_{b_{1} g_{2}} & t_{b_{1} b_{1}} & t_{b_{1} b_{2}} \\
t_{b_{2} r_{1}} & t_{b_{2} r_{2}} & t_{b_{2} g_{1}} & t_{b_{2} g_{2}} & t_{b_{2} b_{1}} & t_{b_{2} b_{2}}
\end{array}\right] \simeq\left[\begin{array}{lllllll}
T_{r_{1}} & T_{r_{2}} & T_{g_{1}} & T_{g_{2}} & T_{b_{1}} & T_{b_{2}}
\end{array}\right]
$$

Fig. 10. Example linear transformation matrix for a 2Bin RGB quantisation

$t_{x y}$ is the term that specifies how much the input from colour channel $x$ contributes to the output of colour channel $y$. Transformation matrices are formed between the four cameras. Six transformations along with their inverses provide the twelve transformations required to transform objects between the four cameras. As camera calibration is refined, the illumination changes that affected the success of the original correlation methods discussed in [3] and section 4, are reduced. This allows other less coarse quantisation (such as $\mathrm{RGB})$ to be used with improved performance as will be shown.

The six transformation matrices for the four cameras are initialised as identity matrices assuming a uniform colour response between cameras. When a person is tracked inter camera and identified as possibly the same object, the transformation between the two colour descriptors is calculated. The matrix $t$ is calculated by computing the transformation that maps the person's descriptor from the previous camera $I$ to the person's current descriptor $T$. This transformation is computed via SVD and weighted by the objects colour 
similarity. The matrix $t$ is then averaged with the appropriate camera transformation matrix, and repeated as people are tracked between cameras to gradually build a colour transformation between the cameras. As not all object correspondences will be true correspondences, this method will introduce small errors. However, it is in keeping with the incremental theme of the paper and again relies upon the fact that given time, statistical trends in the data will emerge. This allows the system to continually update and adapt to the colour changes between cameras as more data becomes available.

To form the transform matrices a number of different quantisations were examined. A 3x3 matrix of the median colour of a person, was found to be too coarse, discarding too much colour information. The 11 bin CCCM quantisation is equally coarse and therefore with calibration quantised RGB performs best. A number of RGB quantisation levels were investigated with varying accuracy, however using In-Parzen-Windowing gives a stable accuracy over a range of quantisation levels.

\section{Experimental Results}

This section demonstrates the performance of the techniques proposed for tracking objects across uncalibrated non overlapping cameras. The final system starts uncalibrated with no a priori information about its environment. It automatically incorporates information as it becomes available over time. The experimental data was accumulated from 9am for 3 days (72 hours), tracking a total of 7854 objects. Evaluation of the tracking was performed using two separate unseen ground-truthed 20 minute sequences each with 200 instances of people tracked for more than 1 second. The two video sequences are quite different (see figure 11 for examples of objects);

- Test Video1, This has a large number of new unique people, people walking in groups and people losing their track intra camera due to erratic and slow movement.

- Test Video2, This consists of more people moving cross camera, with fewer new unique people.

Initially, the system tracks using only the CCCM colour similarity between objects. Objects are tracked by maximising the posterior probability within a set time window, $T$. For these experiments, $T$ is 40 seconds, where the cameras are close, though it could be increased with no effect, provided the maximum peak exceeds the noise floor. While incrementally learning the inter camera relationships of the previously discussed weak cues, the system goes through a number of region size subdivisions. The first possible subdivision is after 1 
Video1
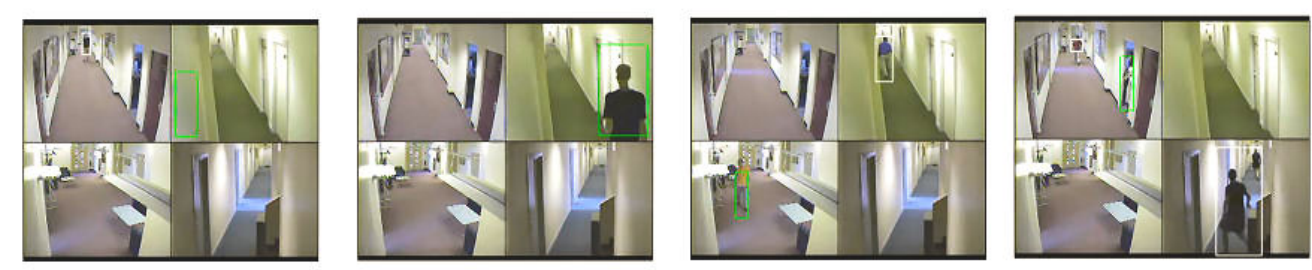

Video2
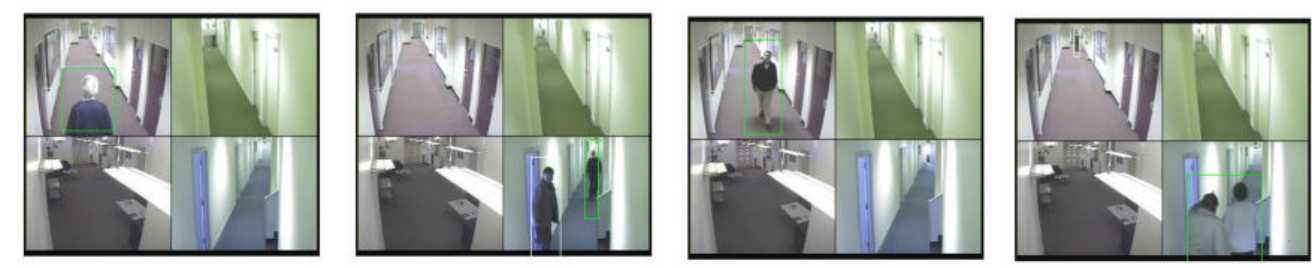

Fig. 11. An example of some of the detected objects from both videos. The box indicates a tracked object

hour, the next after 4 hours of operation, the third division is after 8 hours which corresponds to 1 full working day from 9 am-5pm. The final possible subdivision level is reached after 32 hours which is two full working days. After 56 hours (three full working days) no further subdivisions take place but additional data is continually added to the prior until 72 hours (three full days) has passed. At each stage the accuracy of all techniques of tracking; CCCM colour alone, posterior region links, posterior bounding box, and calibrated RGB colour, are measured. Table 2 shows all the single cues across the region subdivision sizes. The abbreviations for the similarity measures used are:

- HI(CCCM) - Histogram intersection of the CCCM colour descriptor.

- Reg - Maximising the posterior probability using the incrementally learnt prior on reappearance period.

- BB - Maximising the posterior probability using the learnt prior on object exit and entry size.

- HI(RGB) - Histogram intersection of the colour calibrated quantised RGB colour descriptor.

- Batch - Comparison technique of reappearance period prior computed using entry and exit regions derived through a batch processed K-means method.

Table 2 shows the initially poor performance of the individual descriptors. Over time accuracy improves, reaching $65 \%$ for Video 2 using calibrated RGB after one working day or 8 hours. Note that after 8 hours, the priors are relatively stable and little benefit is gained from the addition of a further 2 days worth of observations.

At each stage of region refinement the accuracy of most techniques increases. After 72 hours and 7854 objects, each camera region has been subdivided at 
Table 2

Table of results of using the individual descriptors with no fusion with subdivision of regions as more data is accumulated with up to three days of data

\begin{tabular}{|c|c|c|c|c|c|c|c|c|}
\hline \multirow[t]{2}{*}{ Video } & \multirow[t]{2}{*}{ Method } & \multicolumn{7}{|c|}{ Accuracy: } \\
\hline & & Initial & Sub1 & Sub2 & Sub3 & Sub4 & Sub4 & Sub4 \\
\hline & Time (Hr) & 0 & 1 & 4 & 8 & 32 & 56 & 72 \\
\hline & Data (People) & 0 & 367 & 1372 & 2694 & 5264 & 7612 & 7854 \\
\hline \multirow{4}{*}{ Video1 } & $\mathrm{HI}(\mathrm{CCCM})$ & $50 \%$ & $50 \%$ & $50 \%$ & $50 \%$ & $50 \%$ & $50 \%$ & $50 \%$ \\
\hline & Reg & & $33 \%$ & $41 \%$ & $45 \%$ & $45 \%$ & $44 \%$ & $44 \%$ \\
\hline & $\mathrm{BB}$ & & $42 \%$ & $49 \%$ & $55 \%$ & $58 \%$ & $60 \%$ & $60 \%$ \\
\hline & HI(RGB) & $32 \%$ & $45 \%$ & $51 \%$ & $53 \%$ & $53 \%$ & $55 \%$ & $57 \%$ \\
\hline \multirow{4}{*}{ Video2 } & $\mathrm{HI}(\mathrm{CCCM})$ & $47 \%$ & $47 \%$ & $47 \%$ & $47 \%$ & $47 \%$ & $47 \%$ & $47 \%$ \\
\hline & Reg & & $33 \%$ & $40 \%$ & $51 \%$ & $51 \%$ & $51 \%$ & $52 \%$ \\
\hline & $\mathrm{BB}$ & & $58 \%$ & $64 \%$ & $64 \%$ & $64 \%$ & $64 \%$ & $64 \%$ \\
\hline & HI(RGB) & $40 \%$ & $58 \%$ & $62 \%$ & $65 \%$ & $65 \%$ & $66 \%$ & $67 \%$ \\
\hline
\end{tabular}

most 4 times, with figure 12 showing the main entry/exit areas discovered by the system. Most traditional methods of tracking objects inter camera, [8]

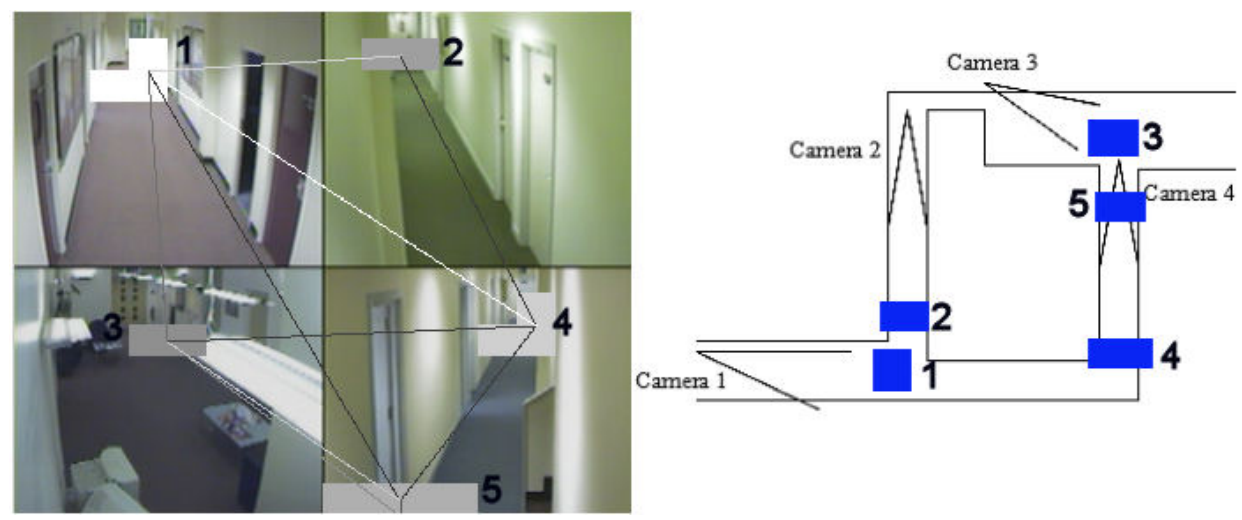

Fig. 12. The main discovered entry and exit regions and a top down layout of the camera system environment with these regions marked

[14], use a batch learning process to discover areas of interest, rather than incremental learning. We include a batch process technique within these results, to compare the effects on performance. After data collection, K-means is performed with 5 components on each camera to cluster the entry/exit positions ( see figure 13). One disadvantage of the batch technique is that there is no accuracy improvement until all data is collected, while the use of an incremental learning algorithm allows the system to increase accuracy over time as more data is collected. This also makes the proposed incremental technique more resistant to environmental changes as changes are incorporated over time. The 
entry/exit regions resulting from the batch K-mean clustering technique can be seen in figure 13, the larger the circle the more important the region. It can be seen that the incrementally learnt regions are very similar is position and importance to that learnt with all the data, batch.

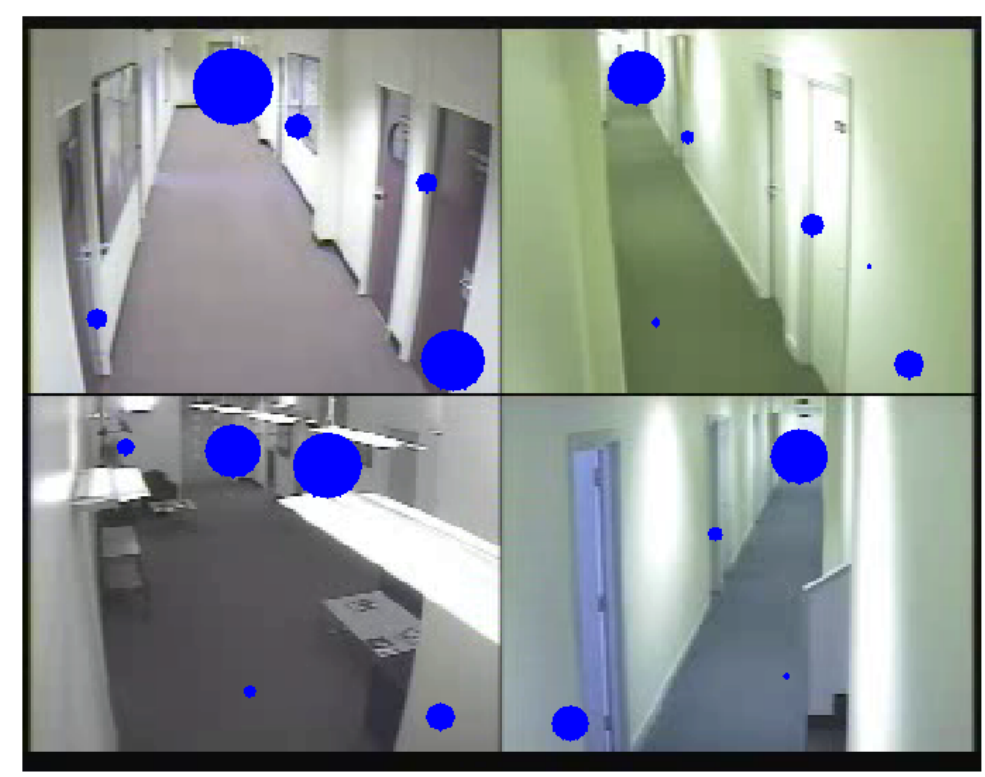

Fig. 13. The main entry and exit regions computed using a batch technique, size of the circles indicates the importance of entry/exit regions

In order to increase the accuracy of inter camera tracking, we can fuse different descriptors together by multiply the likelihoods as discussed in section 6 . This helps to remove some of the limitations. Table 3 shows the results of fusion over the same time period and subdivision intervals as Table 2. Some descriptors are not shown such as $\mathrm{BB}^{*} \mathrm{HI}(\mathrm{CCCM})$ as these performed worse than its colour calibrated equivalent $\mathrm{BB}^{*} \mathrm{HI}(\mathrm{RGB})$ which is shown.

Table 3 shows that the tracking accuracy has been increased from $50 \%$ to $71 \%$ and $47 \%$ to $79 \%$ on video 1 and video 2 respectively when using all three cues (BB*Reg*HI(RGB)) and 8 hours of data (1 working day). Combining all three weak cues together improves accuracy as it removes some of the limitations of each. Table 3 also shows that accumulating a further 2 days of data provides little improvement in accuracy over the cues constructed after only a day. Demonstrating quick convergence upon a good solution. However, as data is accumulated, the posterior match becomes more accurate and this can be used to provide a more accurate match for the calculation of the priors. Table 4 uses one working day of data with the system iterated three times. Each iteration results in an increase in accuracy allowing less false positive correla- 
Table 3

Table of results of using fusing the individual descriptors to increase tracking accuracy from system start-up up to a total of 3 days of data

\begin{tabular}{|c|c|c|c|c|c|c|c|c|}
\hline \multirow[t]{2}{*}{ Video } & \multirow[t]{2}{*}{ Method } & \multicolumn{7}{|c|}{ Accuracy: } \\
\hline & & Initial & Sub1 & Sub2 & Sub3 & Sub4 & Sub4 & Sub4 \\
\hline & Time (Hr) & 0 & 1 & 4 & 8 & 32 & 56 & 72 \\
\hline & Data (People) & 0 & 367 & 1372 & 2694 & 5264 & 7612 & 7854 \\
\hline \multirow{7}{*}{ Video1 } & $\mathrm{HI}(\mathrm{CCCM})$ & $50 \%$ & $50 \%$ & $50 \%$ & $50 \%$ & $50 \%$ & $50 \%$ & $50 \%$ \\
\hline & Reg*HI(CCCM) & & $50 \%$ & $55 \%$ & $62 \%$ & $61 \%$ & $62 \%$ & $64 \%$ \\
\hline & Reg*HI(RGB ) & & $55 \%$ & $64 \%$ & $68 \%$ & $69 \%$ & $69 \%$ & $69 \%$ \\
\hline & BB*Reg & & $49 \%$ & $52 \%$ & $55 \%$ & $56 \%$ & $56 \%$ & $58 \%$ \\
\hline & BB*HI(RGB) & & $59 \%$ & $63 \%$ & $67 \%$ & $67 \%$ & $67 \%$ & $69 \%$ \\
\hline & BB*Reg*HI(RGB) & & $57 \%$ & $62 \%$ & $71 \%$ & $71 \%$ & $73 \%$ & $73 \%$ \\
\hline & Batch & $50 \%$ & $50 \%$ & $50 \%$ & $50 \%$ & $50 \%$ & $50 \%$ & $67 \%$ \\
\hline \multirow{7}{*}{ Video2 } & $\mathrm{HI}(\mathrm{CCCM})$ & $47 \%$ & $47 \%$ & $47 \%$ & $47 \%$ & $47 \%$ & $47 \%$ & $47 \%$ \\
\hline & Reg*HI(CCCM) & & $60 \%$ & $62 \%$ & $72 \%$ & $73 \%$ & $74 \%$ & $75 \%$ \\
\hline & Reg*HI(RGB ) & & $64 \%$ & $66 \%$ & $74 \%$ & $75 \%$ & $77 \%$ & $77 \%$ \\
\hline & BB*Reg & & $55 \%$ & $57 \%$ & $65 \%$ & $66 \%$ & $66 \%$ & $66 \%$ \\
\hline & $\mathrm{BB}^{*} \mathrm{HI}(\mathrm{RGB})$ & & $66 \%$ & $72 \%$ & $74 \%$ & $76 \%$ & $78 \%$ & $78 \%$ \\
\hline & BB*Reg*HI(RGB) & & $66 \%$ & $72 \%$ & $78 \%$ & $79 \%$ & $79 \%$ & $79 \%$ \\
\hline & Batch & $47 \%$ & $47 \%$ & $47 \%$ & $47 \%$ & $47 \%$ & $47 \%$ & $76 \%$ \\
\hline
\end{tabular}

tion to corrupt the three cues. However it can be seen that benefits from this iterative refinement again quickly stabilise. The results of batch learnt links using k-means was combined with histogram intersection on CCCM (labelled Batch in the figure) and can therefore be directly compared with the results of Reg*HI(CCCM) in Table 3. Here it can be seen that batch learning only gives a marginal benefit over the incremental learning scheme. However, this slight increase in performance is only gained at subdivision 4 after 72 hours when all the data is available while the incremental scheme provide a gradual increase in performance as data is acquired.

Table 4, iteration 4 then shows the results of the system after the improvements of the earlier iterations along with the extra data from all 3 days and provides only marginal improvements. These methods improve accuracy due to the minimisation of incorrect matches in the forming of region links and the other cues. This provides a final accuracy of $83 \%$ without data being added, or a slight increase of $85 \%$ if two more days of data is used. Therefore using 
Table 4

Table of results of three iterations of the system after one day of data, but with no new data collected. And results of using 3 days of data after iterating and refining the system accuracy

\begin{tabular}{|c|c||c|c|c||c|}
\hline Video & Method & \multicolumn{4}{|c|}{ Accuracy: } \\
\hline \multirow{1}{*}{} & & Iteration1 & Iteration2 & Iteration3 & Iteration4 \\
\hline \multirow{5}{*}{ Video1 } & Time (Hr) & 8 & 8 & 8 & 72 \\
\hline & Data (People) & 2694 & 2694 & 2694 & 7854 \\
& HI(CCCM) & $50 \%$ & $50 \%$ & $50 \%$ & $50 \%$ \\
& Reg*HI(CCCM) & $64 \%$ & $64 \%$ & $64 \%$ & $66 \%$ \\
& Reg*HI(RGB ) & $71 \%$ & $72 \%$ & $74 \%$ & $73 \%$ \\
& BB*HI(RGB) & $55 \%$ & $57 \%$ & $59 \%$ & $58 \%$ \\
& BB*Reg*HI(RGB) & $73 \%$ & $70 \%$ & $75 \%$ & $77 \%$ \\
\hline \multirow{7}{*}{ Video2 } & HI(CCCM) & $47 \%$ & $47 \%$ & $47 \%$ & $47 \%$ \\
& Reg*HI(CCCM) & $72 \%$ & $72 \%$ & $72 \%$ & $75 \%$ \\
& Reg*HI(RGB ) & $78 \%$ & $79 \%$ & $79 \%$ & $77 \%$ \\
& BB*Reg & $66 \%$ & $65 \%$ & $67 \%$ & $66 \%$ \\
& BB*HI(RGB) & $74 \%$ & $77 \%$ & $77 \%$ & $78 \%$ \\
& BB*Reg*HI(RGB) & $78 \%$ & $83 \%$ & $83 \%$ & $85 \%$ \\
\hline
\end{tabular}

the extra 2 days data gives little or no improvement, again demonstrating the system quickly converges on its maximum after only 8 hours of camera monitoring. Figure 14 gives a visual representation of the accuracy increase over one day of data shown in Tables 2, 3 and further iterations in Table 4. The large increase in initial tracking accuracy from using only colour histogram intersection with the CCCM colour space can be seen. This large increase in accuracy allows the system to fulfil the three ideals stated in the introduction, of working immediately, improving performance as more data is captured, and an ability to adapt to environmental changes.

\subsection{Using the probably to rank the matches in order}

Until now our performance measure was based upon using the top ranked match against the correct person. As each possible correlation returns a likelihood score of a match, these can be presented in a ranked list. This reduces the quantity of data the operator has to process while improving accuracy. 


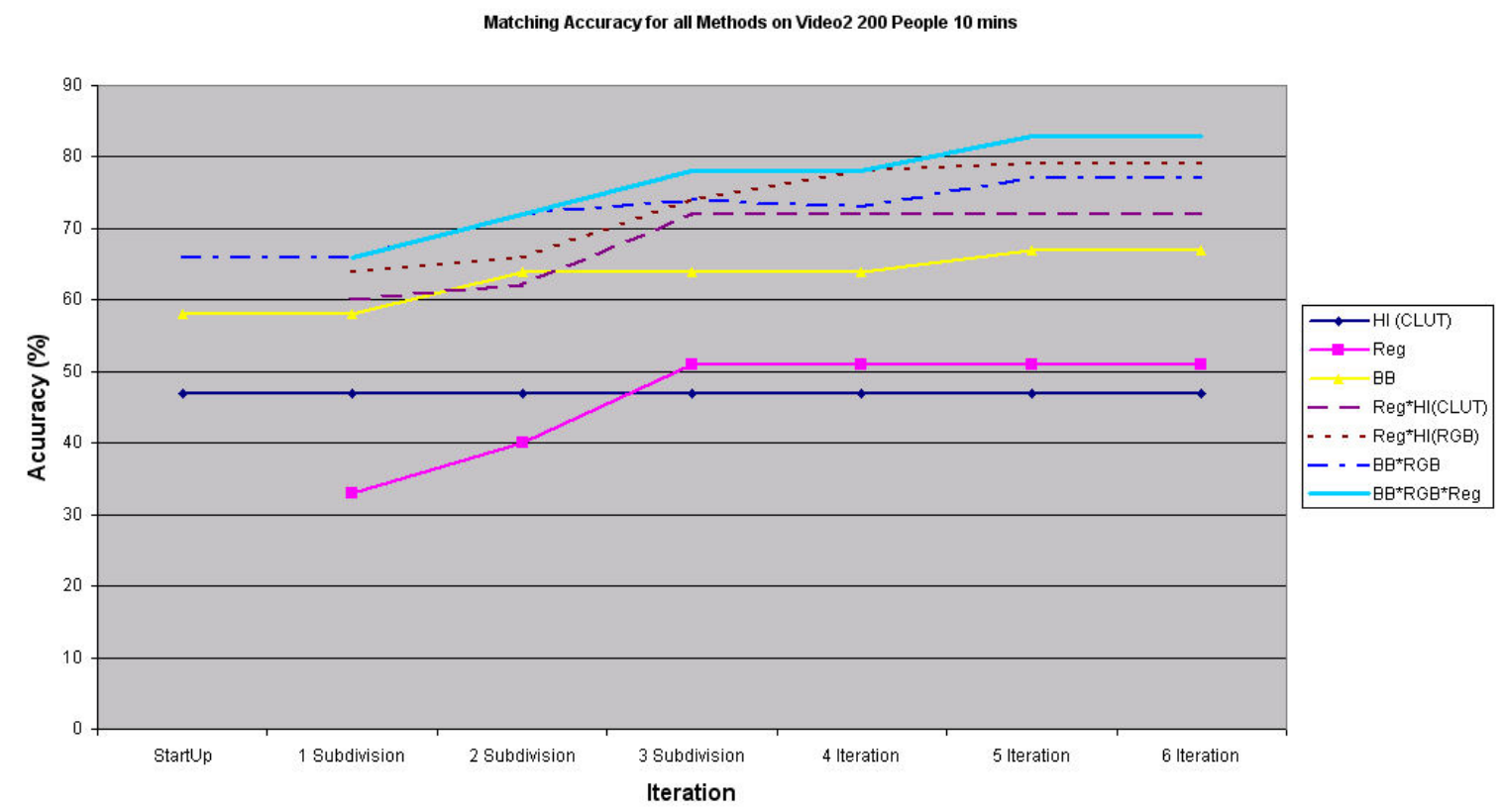

Fig. 14. A graph showing the increasing accuracy with subsequent iterations of the methods using Video2 up to iteration3

An example of this is in figure 15, here the correct match is the third ranked. As all three results have a very similar appearance with likelihoods, 0.15, 0.13 and 0.12 this indicates to the user that there is some uncertainty in the system. This uncertainly is partly responsible for the ceiling accuracy of $85 \%$. However, by considering the top three ranked correlation's, the effective performance can be considered considerably higher. The graph in figure 16, shows the result of this, based on the fusion of the bounding box, region links and histogram intersection of the RGB model. The incoming video stream is the top left image, with the lower left image showing the current query object. On the right are three ranked matches. Scoring using the top 3 ranked matches increases accuracy to over $90 \%$.

\section{Scalability}

This section discusses design considerations for large surveillance systems and how the proposed method can be scaled up. As the number of cameras increase in the system, the architecture of the system and data communication between modules become an important consideration. Traditional systems are based on a client serve architecture. With the server receiving and processing all the video feeds. Communication between cameras is then carried out within the server ensuring high speed. However, as all processing is performed by the single core server, should the server fail, the whole system would be immo- 


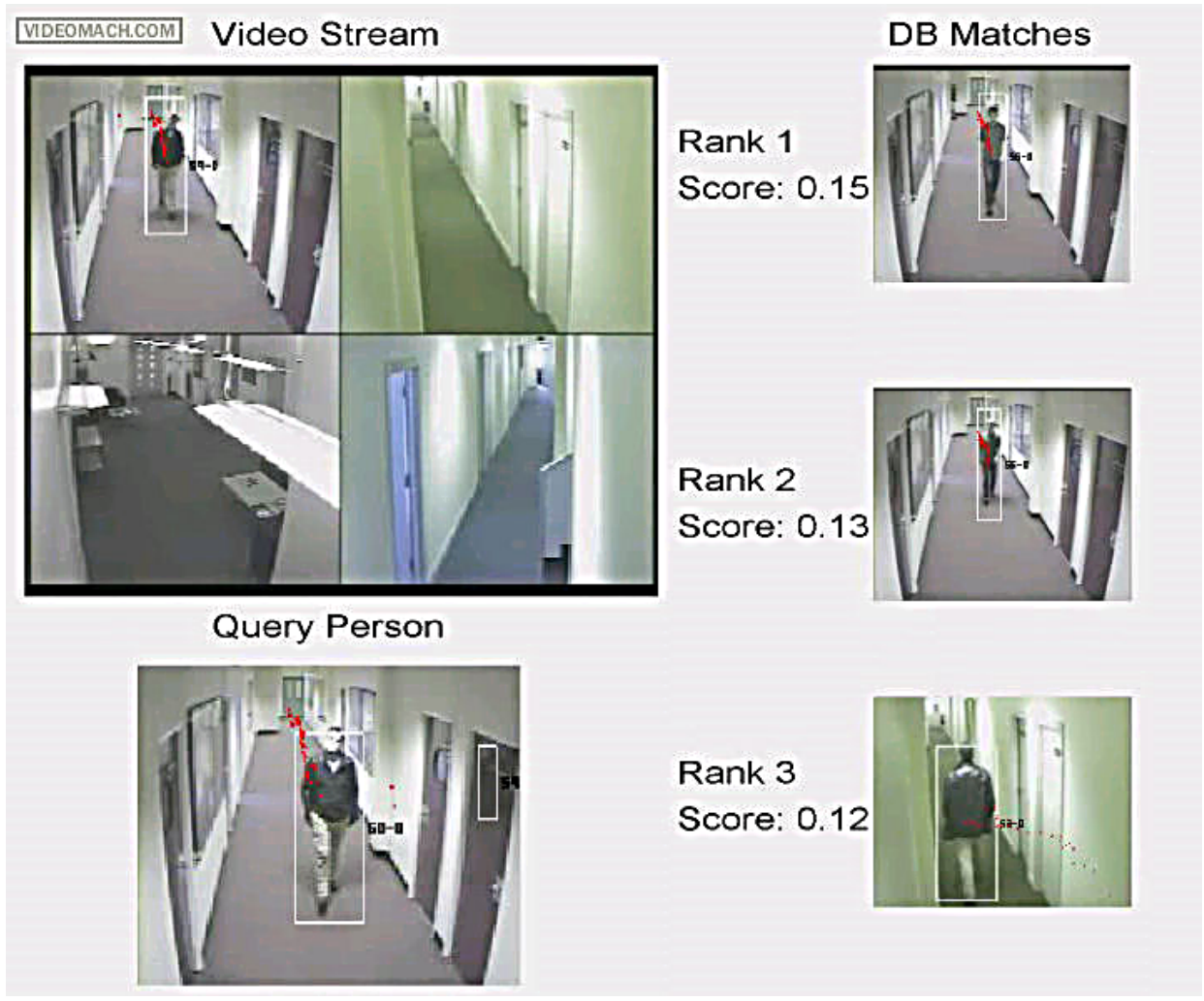

Fig. 15. The system gives a rank of the best matches to the operator instead of the single optimum

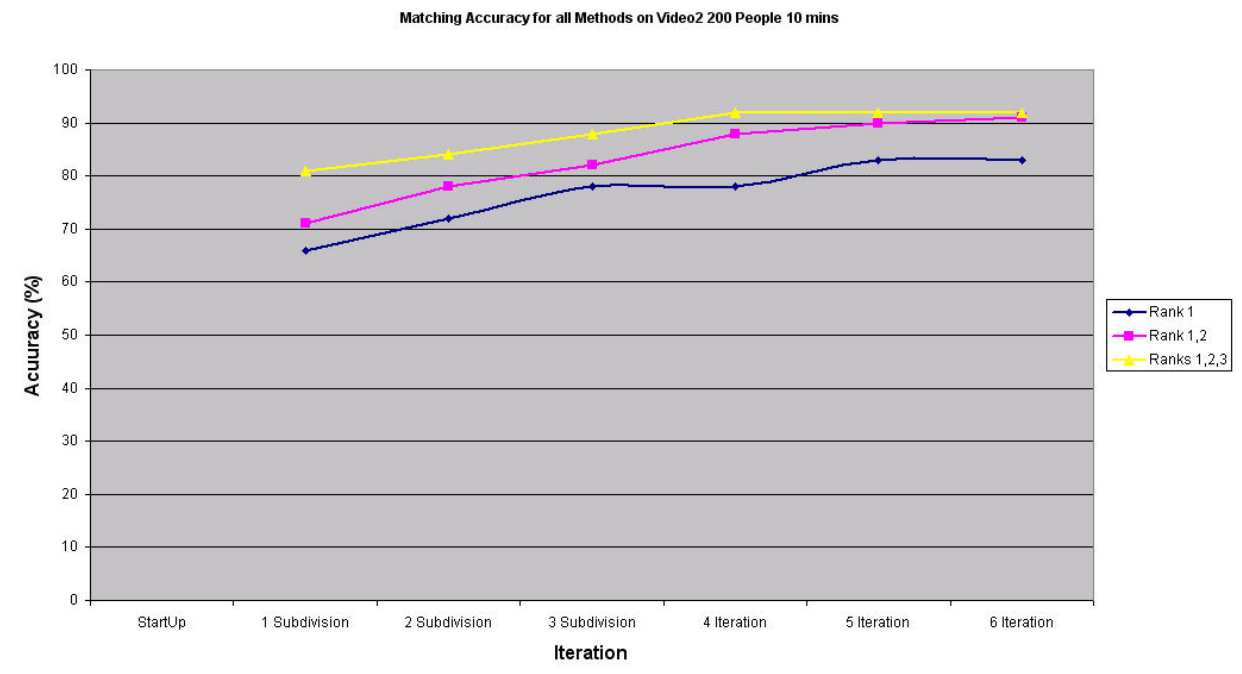

Fig. 16. Using ranked matches to improve accuracy of tracking

bilised. More importantly, the system would be limited by the processing speed of the server, and adding further cameras, would slow the overall performance.

An alternative on which our system is based is a decentralised system which 
operates as a Peer-to-Peer network. In a Peer-to-Peer architecture, there are no servers or clients, but only equal peer nodes that function simultaneously as both "clients" and "servers" to the other camera nodes on the network.

\subsection{Scaleable Learning and Tracking}

The main bottleneck of a peer-to-peer implementation is the increased bandwidth requirements between camera nodes. Therefore, the minimum amount of communication between cameras is essential. When a person is detected on a camera, they will be tracked within the camera while visible. As the object exits the camera, their descriptor and the leaving position is broadcast to all other cameras. All cameras then receive and store this in their short-term memory. As each camera doesn't record the level of region subdivision at the destination of its links, a formalised labelling of the region link is used.

The system is based on a rectangular region subdivision where each camera is divided into 16 regions. To allow for a scaleable system, each region has a formalised 4 digit number which corresponds to the level of subdivision the region has undergone and its originating camera. Initially the system starts with one region per camera. This allows immediate tracking with the links initially uniformly distributed. Figure 17 shows how the subdivision takes place for camera $\mathrm{X}$, with the region ID adding an additional digit for each subdivision. At the first level of subdivision, a single digit is used, then when

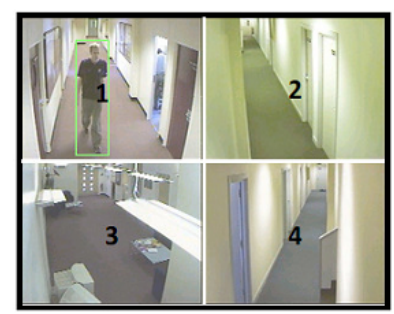

(c) Level 1 Regions
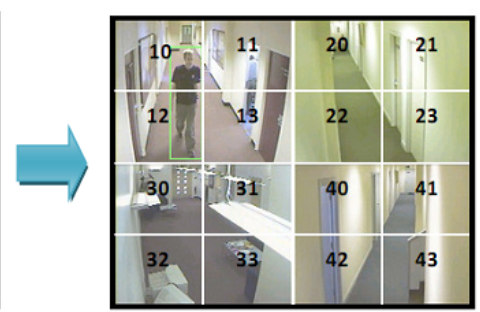

(b) Level 2 Regions

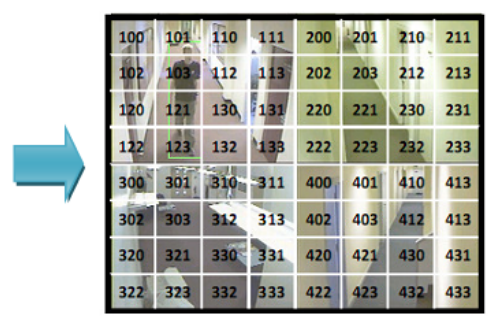

(a) Level 3 Regions

Fig. 17. The 3 levels of region subdivision with their associated numbering. The star indicates a highlighted region and its ID below. (a) shows the initial camera regions, (b) after 1 sub division and (c) after two possible subdivisions.

subdivided another digit is added ()figure 17b). This means that the complete ID for each region also contains the ID for the higher level regions, i.e. the ID X113, says that region 113 is part of the region 11 at a higher level which in turn is part of the region 1 on camera X. This means that links between two cameras can be constructed at the highest resolution support by both cameras. In the example of figure 18, a person has just left camera 1, allowing camera 1 to broadcast their descriptor, along with the exit region which is 
13, making the region ID 113 to all other cameras. A new person appears on camera 2 and will use the descriptor from 113 to the new region (211) on its camera as required. However, when another person is detected on Camera 3, the region on camera 3 has a link to camera 1 at a less detailed level due to lack of data. Therefore camera 3 will use the link between region 1 on camera 1 and region 2 on camera 3. Both cameras 2, and 3 use the same formalised region ID despite them having links to camera 1 that were at varying levels of detail.

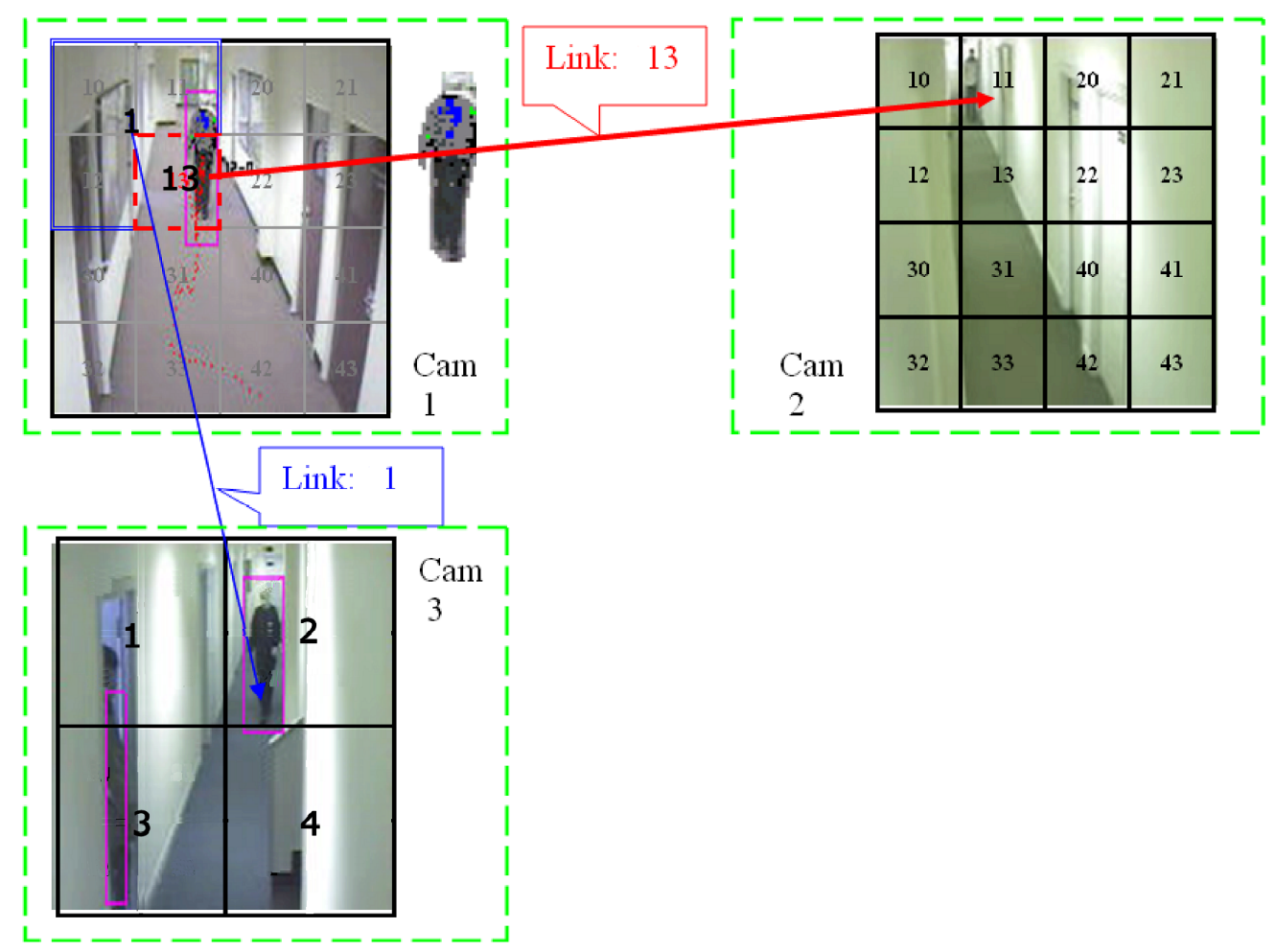

Fig. 18. Example of the scalable region linking based around a formalised region ID system with the person in camera 1 linked to cameras 2 and 3 . With a detailed link to camera 2 from region 13 to region 11, and a less detailed link to camera 3 from region 1 to region 22

For an operator to track a specific object, the operator communicates with the camera the object is currently on, setting a tracking flag. Then as this objects leaves the camera, it is broadcast with the tracking flag. When another camera finds a correlation to the flagged object, the operator is informed of the event without further communication to the original camera. Other cameras that find further correlation within the time threshold also inform the operator about the matches. This allows the operator to make a decision from the top ranked matches returned. 


\section{Conclusion}

We have described an approach to automatically derive three weak cues that describe the relationship between uncalibrated cameras. The main entry and exit regions in a camera are incrementally learnt probabilistically, while simultaneously both the entry and exit size and colour variations inter cameras are also learnt. Together, these are used to overcome the limitations of colour similarity alone (50\%) boasting the accuracy up to $83 \%$ after one day or $85 \%$ after a full three days. This is all carried out on a system that is initially uncalibrated, and has no a priori information about its environment, colours, or objects tracked. The colour observation likelihood is incrementally calibrated to model inter camera colour variations, this is then weighted by both the spatio-temporal region links of the cameras, and the probabilistic size at entry and exit of the cameras. We have demonstrated this approach working on two separate ground-truthed indoor videos. The system can run for many days while building and learning inter camera relationships, while over time becoming more accurate as the model is refined

\section{Acknowledgements}

This work is supported by an EPSRC/Imaging Faraday partnership industrial CASE award sponsored by Overview Ltd.

\section{References}

[1] J. Annesley and J. Orwell. "On the Use of MPEG-7 for Visual Surveillance". 6th IEEE International Workshop on Visual Surveillance, May, Graz, Austria, 2006.

[2] J. Black, T. Ellis, and D. Makris. "Wide Area Surveillance with a MultiCamera Network". Proc. IDSS-04 Intelligent Distributed Surveillance Systems, pages $21-25,2003$.

[3] R. Bowden, A. Gilbert, and P. KaewTraKulPong. "Tracking Objects Across Uncalibrated Arbitrary Topology Camera Networks, in Intelligent Distributed Video Surveillance Systems". S.A Velastin and P Remagnino Eds. Chapt 6, IEE, London, pages 157-183, 2005.

[4] Q. Cai and J. Agrarian. "Tracking Human Motion using Multiple Cameras". Proc. International Conference on Pattern Recognition, pages 67-72, 1996. 
[5] T. Chang and S. Gong. "Bayesian Modality Fusion for Tracking Multiple People with a Multi-Camera System". Proc. European Workshop on Advanced Video-based Surveillance Systems, 2001.

[6] A. Dick and M. Brooks. "A Stochastic Approach to Tracking Objects Across Multiple Cameras". Australian Conference on Artificial Intelligence, pages 160170,2004 .

[7] N. Dowson and R. Bowden. "A Unifying Framework for Mutual Information methods for use in Non-linear Optimisation". In Proc. of the 9th Euopean Conference of Computer Vision, Gratz, Austria, pages 365 -378, 2006.

[8] T. Ellis, D. Makris, and J.K. Black. "Learning a Multi-Camera Topology". Joint IEEE Workshop on Visual Surveillance and Performance Evaluation of Tracking and Surveillance (VS-PETS), pages 165-171, 2003.

[9] M. Felsberg and G. Granlund. "P-Channels: Robust Multivariate M-Estimation of Large Datasets". Proc, ICPR'06, 2006.

[10] A. Gilbert and R. Bowden. "Tracking Objects Across Cameras by Incrementally Learning Inter-Camera Colour Calibration and Patterns of Activity". Proc. ECCV'06, Gratz Austria, page 125 136, 2006.

[11] T. Huang and S. Russell. "Object Identification in a Bayesian Context". Proc. International Joint Conference on Artificial Intelligence (IJCAI-97), Nagoya, Japan, pages 1276-1283, 1997.

[12] A. Ilie and G. Welch. "Ensuring Color Consistency across Multiple Cameras". Techincal Report TR05-011, 2005.

[13] O. Javed, Z. Rasheed, K. Shafique, and M. Shah. "Tracking Across Multiple Cameras with Disjoint Views". Proc. IEEE International Conference on Computer Vision, pages 952-957, 2003.

[14] P. KaewTrakulPong and R. Bowden. "A Real-time Adaptive Visual Surveillance System for Tracking Low Resolution Colour Targets in Dynamically Changing Scenes". Journal of Image and Vision Computing. Vol 21, Issue 10, Elsevier Science Ltd, pages 913-929, 2003.

[15] P. KaewTrakulPong and R. Bowden. "Towards Automated Wide Area Visual Surveillance: Tracking Objects Between Spatially Separated, Uncalibrated Views". In Proc. Vision, Image and Signal Processing, Vol 152, issue 02, pages 213-224, 2005.

[16] P. Kelly, A. Katkere, D. Kuramura, S. Moezzi, and S. Chatterjee. "An Architecture for Multiple Perspective Interactive Video". Proc. of the 3rd ACE International Conference on Multimedia, pages 201-212, 1995.

[17] V. Kettnaker and R. Zabih. "Bayesian Multi-Camera Surveillance". Proc. IEEE Computer Vision and Pattern Recognition, pages 253-259, 1999.

[18] Joshi N. "Color Calibrator for Arrays of Inexpensive Image Sensors". MS Thesis, Stanford University Department of Computer Science, 2004. 
[19] F. Porikli. "Inter-Camera Color Calibration by Cross-Correlation Model Function". IEEE International Conference on Image Processing (ICIP), Vol. 2, pages 133-136, 2003.

[20] GretagMacbeth Color Management Solutions. www.gretagmacbeth.com.

[21] C. Stauffer and W.E.L. Grimson. "Learning Patterns of Activity using Realtime Tracking". PAMI, 22(8), pages 747-757, 2000.

[22] J. Sturges and T.W.A. Whitfield. "Locating Basic Colour in the Munsell Space". Color Research and Application, 20(6):364-376, 1995.

[23] M. Swain and D. Ballard. "Color Indexing.". Int. Journal of Computer Vision, 7(1):11-32, 1991.

[24] G. Welch and G. Bishop. "An Introduction to the Kalman Filter". Technical Report 95-041 University of North Carolina, Department of Computer Science, 1995. 